\title{
David J. Thouless (1934-2019)
}

\section{Theoretical physicist who discovered topological phases of matter.}

$\mathrm{D}$ avid Thouless was best known for his work on what drives the rearrangement of the constituents of systems, such as the atoms in a crystal or the spins associated with the electrons in a magnet. These 'ordering phenomena' underlie phase transitions, for example when water becomes ice.

In the early 1970s, Thouless predicted a particular type of ordering, along with his colleague Michael Kosterlitz. The Kosterlitz-Thouless transition has since been observed in superfluids, superconductors, magnets and other two-dimensional quantum systems. It was largely for this contribution that Thouless and Kosterlitz shared a physics Nobel prize in 2016 with another theorist, Duncan Haldane.

A deep and original thinker, Thouless made many significant contributions, in areas as wide-ranging as nuclear matter, statistical mechanics and condensed-matter physics. A theme of much of his work was topology, and how it can lead to non-trivial configurations within the constituent parts of a system. Over the past decade, the realization that certain topological features, such as vortices and knots, can arise in the arrangement of electrons in many materials has caused a burst of interest in what are known as topological states of matter. Researchers aim to use these as building blocks for applications in electronics, such as making computing more energy efficient. This exciting area is the direct offspring of Thouless's ideas.

Born in Bearsden, UK, Thouless grew up in Cambridge. Both parents were academics: his mother lectured in English literature; his father, psychology. Thouless's aptitude for mathematics was recognized early, and his father instilled in him a deep curiosity and independence of thought that characterized his career.

After attending Winchester College on a scholarship, Thouless studied natural sciences at the University of Cambridge. Here, he met the future Nobel prizewinner Hans Bethe, then on sabbatical at the Cavendish Laboratory. Bethe invited him to do a PhD at Cornell University in Ithaca, New York. So, in 1956, Thouless set sail for the United States. His experience at Cornell was a happy one. He worked on the application of perturbation methods to the theory of nuclear matter, and met his future wife, Margaret Scrase.

Next came a stint at the Lawrence Radiation Laboratory in Berkeley, California. In

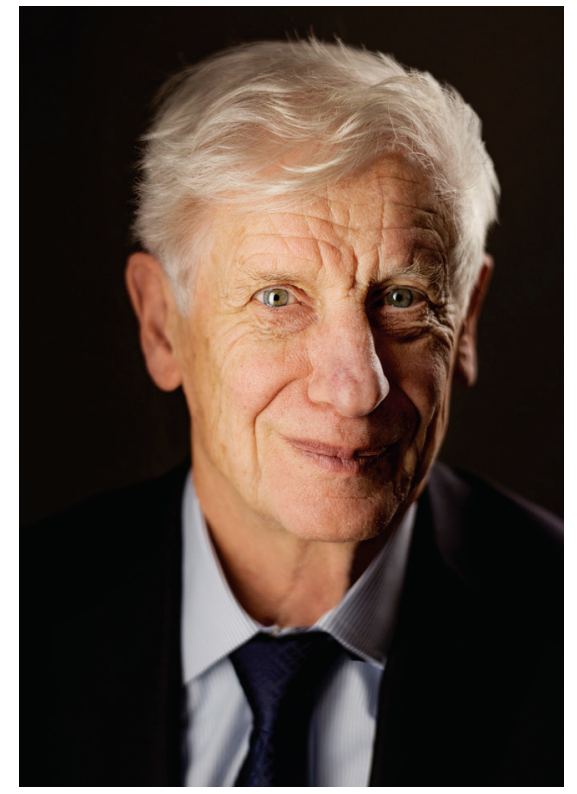

1959, Thouless returned to the United Kingdom for a postdoctoral fellowship at the University of Birmingham, to work on the collective motion of nuclei. There, he experienced what he later described as the most interactive time of his career. $\mathrm{He}$ worked under his mentor, Rudolf Peierls, whose open approach and broad connections (forged during the Manhattan Project) helped to cultivate a world-class Department of Mathematical Physics. Birmingham is also where - a decade later - Thouless carried out his most famous work.

Following a few years as a lecturer in Cambridge, Thouless was appointed professor at Birmingham in 1965. During this period, his research interests shifted towards statistical mechanics - especially the study of phase transitions, a field that was undergoing a revolution at the time.

A typical phase transition might involve, say, a magnetic material switching to a non-magnetic state above a certain critical temperature. However, a class of $2 \mathrm{D}$ systems (known as continuous spin systems) presented a conundrum. A rigorous theorem ruled out the stability of an ordered, magnetic phase at low temperature. Yet various investigations - theoretical, numerical and experimental - suggested the presence of a phase transition.

How could this discrepancy be resolved? The answer lay in the existence of vortices, topological excitations that had hitherto been largely overlooked. Kosterlitz and Thouless realized that, at low temperatures, a topologically ordered phase existed in which interacting pairs of vortices are thermodynamically stable. At higher temperatures, these vortices unbind and move away from each other, and are eventually destroyed by the system's entropy.

Independently, Kosterlitz followed up this work with a more rigorous theoretical derivation. More importantly, perhaps, other research groups were able to demonstrate the Kosterlitz-Thouless transition experimentally. The most famous of these demonstrations, carried out by David Bishop and John Reppy in the late 1970s, used thin films of superfluid helium. But the beauty of the vortex-unbinding mechanism is its universality. The same ideas can be used to describe the behaviour of ultrathin superconductors and magnets, for example, as well as the melting of a $2 \mathrm{D}$ crystal.

In 1980, Thouless joined the University of Washington in Seattle, where he thrived until his retirement, along with his wife, who held a faculty position in pathobiology there. In another profound study that was cited by the Nobel committee, he helped to expose the topological origin of the quantum Hall effect, the stepwise increase in electrical conductance seen in ultrathin conducting layers at low temperatures. This work established the conceptual framework that would eventually allow topological insulators to be discovered.

Indeed, Thouless's 1998 book, Topological Quantum Numbers in Nonrelativistic Physics, which brought together many of the themes that had interested him throughout his career, is notable for its prescience. More than two decades since its publication, it is more relevant to our modern understanding of topological phases of matter than ever.

By his own account, David Thouless had an introverted personality, preferring to collaborate with colleagues rather than lead a group. Leaps between stages of his powerful arguments left many lost until hours later. But those who were fortunate enough to work with him directly remember him warmly, both for his gentlemanly demeanour and for his brilliant - if economically expressed - scientific insights.

Andrea Taroni is the chief editor of Nature Physics.

e-mail:a.taroni@nature.com;

Twitter: @TaroniAndrea 